\title{
Accuracy of MR Imaging for Detection of Sensorineural Hearing Loss in Infants with Bacterial Meningitis
}

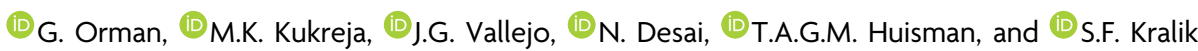

\begin{abstract}
BACKGROUND AND PURPOSE: Bacterial meningitis most commonly affects young children and can result in critical adverse outcomes, including sensorineural hearing loss (SNHL). The purpose of this study is to determine the diagnostic accuracy of MR imaging for predicting the development of SNHL among infants with bacterial meningitis.

MATERIALS AND METHODS: A retrospective review was performed among infants (age <365 days) with bacterial meningitis $(n=115)$. Independent and consensus blinded review of brain MRIs $(n=239)$ performed less than 90 days from presentation were conducted. Abnormal appearance of the inner ear was defined as enhancement on postcontrast T1-weighted (T1-weighted+C) sequence and FLAIR hyperintensity. The consensus MR imaging appearance of the inner ear on FLAIR, T1-weighted+C, and combined evaluation was compared with criterion standard audiometric testing to determine the sensitivity and specificity of MR imaging for detecting SNHL.
\end{abstract}

RESULTS: The mean age at diagnosis of bacterial meningitis was 50.6 days (range, 0-338 days) and 24.3\% had SNHL. Sensitivity and specificity was $0.61 / 0.96,0.50 / 0.94$, and $0.61 / 0.94$ for T-weighted+C, FLAIR hyperintensity, and combined evaluation, respectively, for prediction of SNHL. There was excellent interobserver agreement for both the Ti-weighted $+\mathrm{C}$ and FLAIR sequences and combined evaluation for presence of abnormal enhancement and hyperintense signal, respectively. Factors associated with abnormal MR imaging findings on T7-weighted $+\mathrm{C}$ and/or FLAIR in patients with SNHL included low CSF glucose $(P=.04, .02)$ and high CSF protein $(P=.04, .03)$.

CONCLUSIONS: Abnormal enhancement and/or FLAIR hyperintensity of the inner ear demonstrate high specificity and average sensitivity for prediction of SNHL among infants with bacterial meningitis.

ABBREVIATIONS: $\mathrm{WBC}=$ white blood cell; SNHL = sensorineural hearing loss; GBS = Group B Streptococcus; PPV = positive predictive value; NPV = negative predictive value; $+\mathrm{C}=$ postcontrast

M eningitis is an inflammation of the meninges affecting the pia, arachnoid, and subarachnoid space in response to infection. Despite advances in antimicrobial therapy and improvements in supportive care, bacterial meningitis remains a devastating disease. Group B Streptococcus (GBS) and Escherichia coli (E coli) remain the most common causes of bacterial meningitis in the first 90 days of life. ${ }^{1}$ In the modern era, the mortality of bacterial meningitis in infants is approximately $10 \%$, and survivors remain at high risk for neurologic sequelae. ${ }^{2,3}$

Received November 15, 2019; accepted after revision March 21, 2020.

From the Edward B. Singleton Department of Radiology (G.O., M.K.K., N.D.,

T.A.G.M.H., S.F.K.); and Department of Pediatrics, Section of Infectious

Diseases (J.G.V.), Texas Children's Hospital and Baylor College of Medicine, Houston, Texas.

Please address correspondence to Gunes Orman, MD, Texas Children's Hospital, Edward B. Singleton Department of Radiology, 6701 Fannin St, Suite 470, Houston, TX 77030, e-mail: gxorman@texaschildrens.org

Indicates article with supplemental on-line table.

http://dx.doi.org/10.3174/ajnr.A6539
Sensorineural hearing loss (SNHL) is a serious complication and sequela of bacterial meningitis, occurring in $7 \%-33 \%$ of pediatric patients with bacterial meningitis. ${ }^{4-7}$ SNHL in children is more common with Streptococcus pneumoniae ( $S$ pneumoniae) (31\%) or Neisseria meningitidis ( $N$ meningitidis) (23.9\%) meningitis. ${ }^{5}$

Meningitis leads to SNHL through a presumed mechanism of spread into the inner ear from the CSF through the cochlear aqueduct. ${ }^{8,9}$ Three previous studies have specifically investigated MR imaging for detection of inner ear abnormalities in patients with bacterial meningitis. ${ }^{8-10}$ These studies showed that MR imaging could detect inner ear abnormalities in patients who developed SNHL. However, these studies only included a small number of patients, included a wide range of patient ages, and did not evaluate the value of the FLAIR sequence, a noncontrast MR imaging sequence routinely performed and useful for detecting abnormal fluid. To date, no large study has evaluated both noncontrast and postcontrast MR imaging techniques for prediction of SNHL among 
Table 1: Demographic findings

\begin{tabular}{|c|c|}
\hline Age & 50.6 Days (0-338 Days) \\
\hline Sex & 67 male/48 female \\
\hline \multicolumn{2}{|l|}{ Pathogens } \\
\hline \multicolumn{2}{|l|}{ SNHL(+) } \\
\hline \multirow[t]{5}{*}{ CSF culture results } & Negative (25\%) \\
\hline & GBS (21.4\%) \\
\hline & E coli $(17.9 \%)$ \\
\hline & S pneumoniae $(17.9 \%)$ \\
\hline & $\begin{array}{l}\text { Other pathogens ( } H \text { influenza, } S \text { gallolyticus, } N \text { meningitidis, } \\
S \text { infantarius, and } P \text { mirabilis) (17.9\%) }\end{array}$ \\
\hline \multirow[t]{6}{*}{ Blood culture results } & E coli $(29.6 \%)$ \\
\hline & GBS (22.2\%) \\
\hline & S pneumoniae (14.8\%) \\
\hline & H influenza (7.4\%) \\
\hline & K pneumoniae $(7.4 \%)$ \\
\hline & $\begin{array}{l}\text { Other pathogens (S gallolyticus, } N \text { meningitidis, } S \text { infantarius, } \\
P \text { mirabilis, and } S \text { hominis) (18.5\%) }\end{array}$ \\
\hline \multicolumn{2}{|l|}{ SNHL(-) } \\
\hline \multirow[t]{6}{*}{ CSF culture results } & Negative (30\%) \\
\hline & E coli $(25 \%)$ \\
\hline & GBS (21.3\%) \\
\hline & Other pathogens (Gram-positive cocci, Coccobacilli, \\
\hline & Salmonella, Serratia, S alpha, S gallolyticus, S gamma, \\
\hline & S pneumonia, S pyogenes) (23.7\%) \\
\hline \multirow{5}{*}{ Blood culture results } & GBS $(29.1 \%)$ \\
\hline & Other pathogens (Coagulase negative staphylococcus, \\
\hline & Enterobacter, $H$ influenza, Klebsiella, Neisseria, $S$ aureus, \\
\hline & S bovis, S gallolyticus, S pneumonia, S pyogenes) (24\%) \\
\hline & Negative $(11.4 \%)$ \\
\hline
\end{tabular}

and postcontrast axial and coronal T1-weighted (T1-weighted $+\mathrm{C})$ turbo spin-echo imaging. Section thickness for all FLAIR and T1-weighted $+\mathrm{C}$ sequences was 3-4 $\mathrm{mm}$ with zero gap. MR imaging performed within 90 days of presentation was included.The mean time intervals between meningitis presentation to audiometric testing and between first MRI to audiometric testing were calculated (days). Nondiagnostic MRIs were excluded from the study. Forty-nine patients had $1 \mathrm{MR}$ imaging, and 66 patients had more than $1 \mathrm{MR}$ imaging, of which, 10 patients had differences and 56 patients had no differences on follow-up. Therefore, 239 MRIs of 115 patients were included, of which 239/239 MRIs had the FLAIR sequence and 212/ 239 MRIs had T1-weighted $+\mathrm{C}$ sequences. The 27 MRIs without T1weighted $+\mathrm{C}$ sequences were due to a decision to not administer IV contrast in the setting of renal failure.

Retrospective independent reviews of brain MRIs were performed by 2 infants with bacterial meningitis. In addition, only a limited number of studies have evaluated associated factors that may result in detectable MR imaging abnormalities in these patients such as CSF parameters at the diagnosis or timing of MR imaging in the course of meningitis. Early recognition of labyrinthitis in infants with meningitis by neuroimaging can benefit patient care through promoting earlier audiologic consultation and/or intervention in these patients.

The purpose of this study was to determine the diagnostic accuracy of noncontrast and postcontrast MR imaging techniques for prediction of SNHL among a large cohort of infants with a history of meningitis and the factors associated with abnormal MR imaging findings of the inner ear.

\section{MATERIALS AND METHODS}

Following institutional review board approval, a retrospective review was performed among neonates and infants (age $<365$ days) with confirmed bacterial meningitis imaged between 2011 and 2019. Diagnosis of meningitis was determined by either 1) a positive CSF culture, or 2) positive blood culture combined with elevated CSF white blood cell (WBC) count $(>20 \mathrm{WBC} / \mu \mathrm{L}$ for age $<30$ days, $>9 \mathrm{WBC} / \mu \mathrm{L}$ for age $30-90$ days, and $>6 \mathrm{WBC} / \mu \mathrm{L}$ for age $>90$ days). ${ }^{11,12}$ Patients with immunodeficiency, malignancy, or an intracranial shunt were excluded. The electronic medical records were reviewed to determine patient age at presentation, prematurity ( $<37$ weeks gestation), and audiometric testing results.

MR imaging of the brain was performed using $1.5 \mathrm{~T}$ and $3 \mathrm{~T}$ scanners with precontrast axial and sagittal T1-weighted (T1weighted) turbo spin-echo, axial and/or coronal FLAIR, axial and coronal T2-weighted, axial gradient-echo, axial diffusion-weighted, board-certified pediatric neuroradiologists (S.F.K., M.K.K.) who were blinded to SNHL status, with 7 years and 9 years of experience. Abnormal appearance of the inner ear on MR imaging was defined as presence of abnormal enhancement on T1-weighted $+\mathrm{C}$ sequences (yes/no), or presence of hyperintense FLAIR signal (yes/no). The side of abnormal enhancement or hyperintense FLAIR signal (right/left/bilateral), and subjective degree of enhancement (none/ $\mathrm{mild} /$ moderate/severe) were also recorded. For all patients in whom there was a discordant MR imaging finding, the reviewers performed a combined review, reached a consensus on the MR imaging finding, and this consensus was used as the final diagnosis. Interobserver agreement for individual MR imaging findings was calculated using the $\kappa$ statistic. A $\kappa$ value of $0.81-1.0$ indicated excellent agreement, $0.61-0.80$ indicated good agreement, $0.41-0.60$ indicated moderate agreement, $0.21-0.40$ indicated fair agreement, and $0-0.20$ indicated slight agreement.

Audiometric testing was used as the criterion standard for diagnosis of SNHL in all patients. All audiometric testing was performed by certified audiologists using age-appropriate testing, which included visual reinforcement audiometry, otoacoustic emissions test, auditory brain stem response or auditory steady-state response evaluation. SNHL was graded per hearing levels in decibels $(\mathrm{dB})$ by slight/mild $(26-40 \mathrm{~dB})$, moderate (41$60 \mathrm{~dB})$, severe $(61-80 \mathrm{~dB})$ and profound $(>81 \mathrm{~dB})$ by World Health Organization grades of hearing impairment (https:// www.who.int/pbd/deafness/hearing_impairment_grades/en/).

Sensitivity, specificity, positive predictive value (PPV), and negative predictive value (NPV) of enhancement on T1-weighted $+\mathrm{C}$, FLAIR, and combined evaluation were calculated using the results 


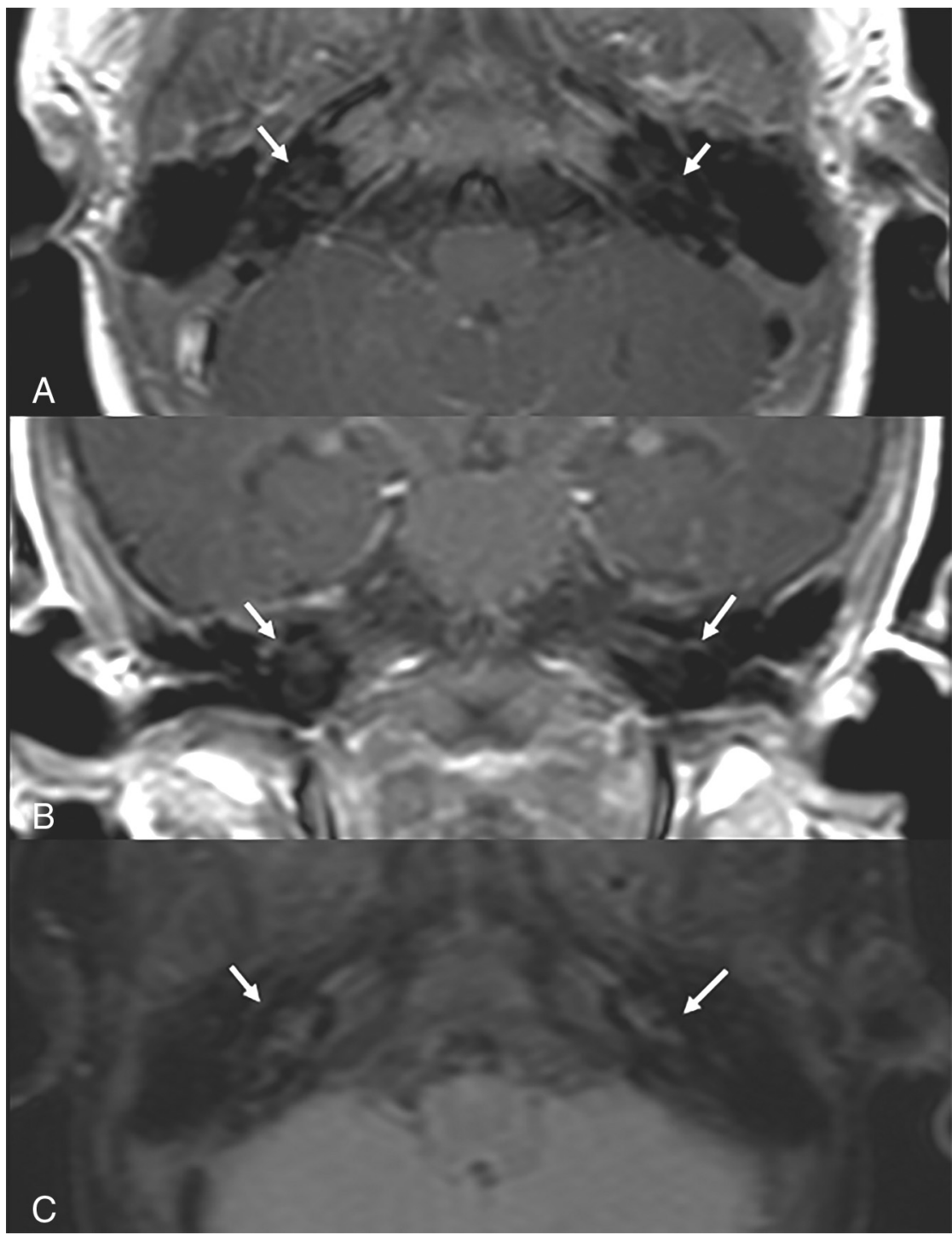

FIG 1. Two-month-old male infant with history of E coli meningitis. Magnified MR imaging of the brain with $(A)$ axial, $(B)$ coronal T1-weighted $+C$, and $(C)$ axial FLAIR reveals normal signal characteristics of the inner ear. The patient did not develop SNHL on follow-up.

(Table 1). The mean time from diagnosis of bacterial meningitis to MR imaging was 16.3 days (range 0-79 days). Mean time from diagnosis of bacterial meningitis to audiometric testing was 323.25 days (range, 0-2268 days). Mean time from the first MR imaging to audiometric testing was 311.7 days (range, 0-2234 days). There was no significant difference in time from the first MR imaging to audiometric testing between infants without SNHL (306.01 \pm 586.9 days) and infants with SNHL (328.5 \pm 469 days) $(P=$ .85). CSF culture and blood culture results for patients with and without SNHL are shown in Table 1.

SNHL was present in $24.3 \%(28 / 115)$ of infants. With respect to age at diagnosis of meningitis, SNHL was $n=12$ (14.6\%) for age $\leq 28$ days, $n=8(34.8 \%)$ for age 29-90 days, and $n=8$ (80\%) for age 90-365 days. In Figs 1-3, the FLAIR and T1-weighted $+\mathrm{C}$ MR imaging findings are shown for patients without and with SNHL. World Health Organization grades of hearing impairment were unilateral slight/mild in $22.2 \%(n=6)$, bilateral slight $/$ mild in $37 \%(n=10)$, bilateral moderate in $7.4 \% \quad(n=2)$, bilateral severe in $7.4 \%(n=2)$, unilateral profound in $11.1 \%(n=3)$, bilateral profound in $14.8 \%(n=4)$ of patients with SNHL. One child had profound SNHL on the right side and slight/mild SNHL on the left side.

A total of $19.8 \%(42 / 212)$ of MR images had consensus interpretation of abnormal enhancement on T1weighted + C. Sensitivity, specificity,

of audiometric testing as the reference standard. Binomial exact 95\% confidence intervals were calculated to provide an estimate of precision. When a patient had more than $1 \mathrm{MR}$ imaging scan before the audiometric testing, the same audiometric testing result was used for the analysis.

To determine the factors associated with an abnormal MR imaging appearance of the inner ear, an unpaired $t$ test was used to compare the age at presentation, time from presentation to MR imaging, CSF WBC count, CSF glucose, and CSF protein. In addition, a Fisher exact test was used to compare positive or negative CSF results between the groups with normal versus abnormal appearance on T1-weighted $+\mathrm{C}$ and FLAIR imaging. A $P$ value $<.05$ was considered statistically significant.

\section{RESULTS}

A total of 115 infants (67 males/48 females) with a mean age of 50.6 days (range, $0-338$ days) met the inclusion criteria for the study
$\mathrm{PPV}$, and NPV of T1-weighted $+\mathrm{C}$ MR imaging findings compared with audiometric testing as the reference standard with 95\% confidence intervals are shown in the On-line Table. Interobserver agreement between the 2 readers was excellent for the presence of abnormal contrast enhancement $(\kappa=0.95)$. The consensus grade of enhancement was $80.9 \%$ mild, $16.7 \%$ moderate, and $2.4 \%$ severe. There was excellent interobserver agreement for the grading of enhancement $(\kappa=0.91)$. The consensus side of the contrast enhancement on T1-weighted $+\mathrm{C}$ was right-sided only $0.9 \%$ (2/212), left-sided only $5.7 \%$ (12/212), bilateral $12.7 \%$ (27/212), and none $80.7 \%(171 / 212)$ for the consensus reading. Interobserver agreement between the 2 readers was excellent for the side of contrast enhancement $(\kappa=0.94)$.

A total of $18.4 \%(44 / 239)$ of MR images had consensus interpretation of hyperintense FLAIR signal. Sensitivity, specificity, PPV, and NPV of FLAIR MR imaging findings compared with audiometric testing as the reference standard with $95 \%$ confidence 


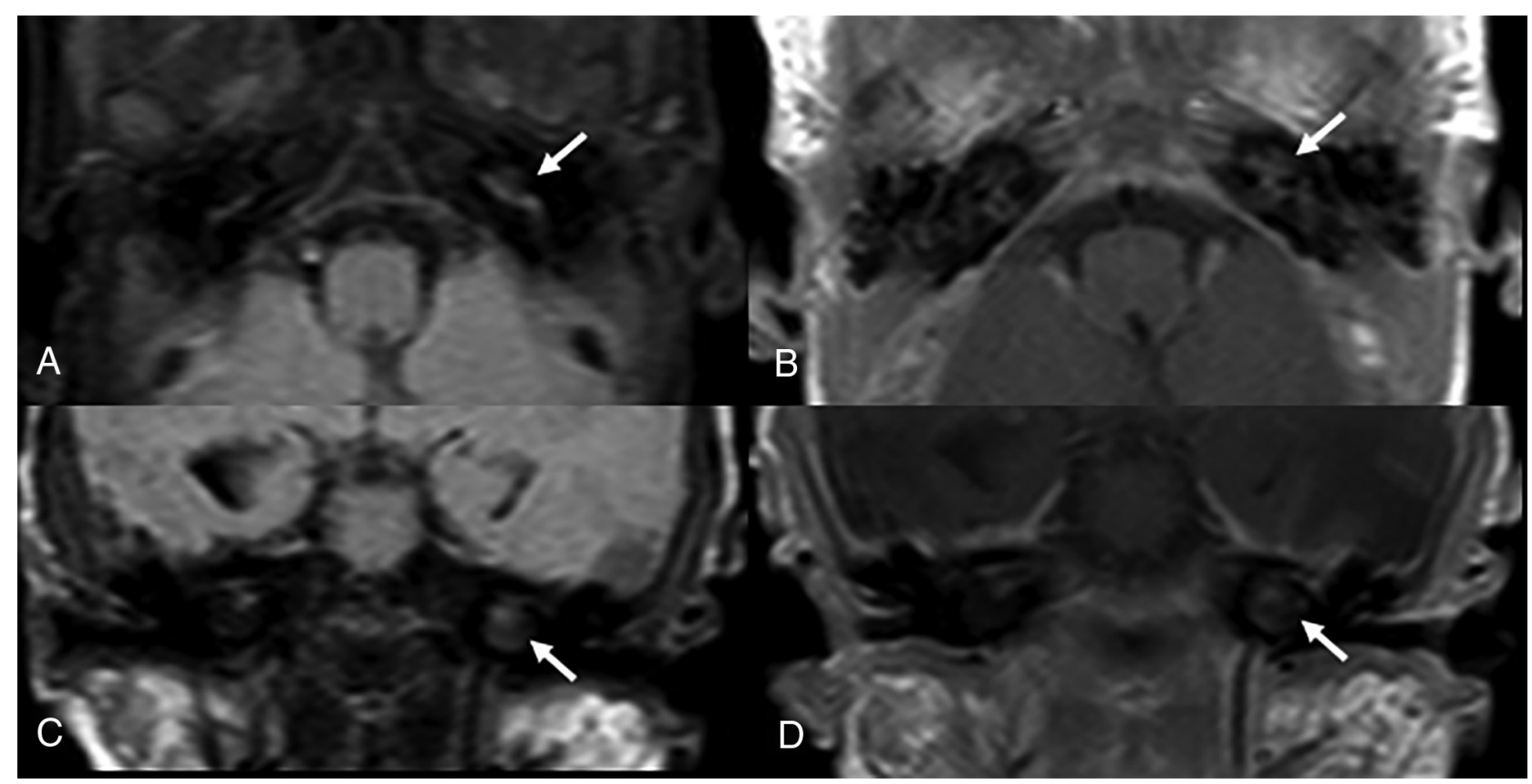

FIG 2. One-month-old male infant with history of late onset GBS meningitis and subsequent cardio-respiratory arrest. Magnified MR imaging of the brain with $(A)$ axial FLAIR, $(B)$ axial T1-weighted $+C,(C)$ coronal FLAIR, and $(D)$ coronal Tl-weighted $+C$ demonstrates abnormal hyperintense FLAIR signal and enhancement of the left inner ear. The patient developed left-sided SNHL on follow-up.

intervals are shown in the On-line Table. Interobserver agreement between the 2 readers was excellent for presence of FLAIR hyperintensity $(\kappa=0.93)$. Side of the FLAIR hyperintensity was rightsided only $0.4 \%(1 / 239)$, left-sided only $3.3 \%$ (8/239), bilateral $14.6 \%$ (35/239), and none $81.6 \%$ (195/239) for the consensus reading. Interobserver agreement between the 2 readers was excellent for the side of abnormal FLAIR hyperintensity $(\kappa=0.90)$.

Among children with SNHL, the mean CSF glucose was significantly lower for abnormal T1-weighted $+\mathrm{C}$, hyperintense FLAIR signal, and combined evaluation ( $P=.04, .02$, and .04 , respectively) and the mean CSF protein was significantly higher for abnormal T1-weighted $+\mathrm{C}$, hyperintense FLAIR signal, and combined evaluation in patients with SNHL $(P=.04, .03$, and .04 , respectively) (Table 2). The presence of any positive CSF culture demonstrated significant association with abnormal T1-weighted $+\mathrm{C}$, hyperintense FLAIR signal, and combined evaluation $(P=.009, .007$, and .003 , respectively). The most common pathogens in patients with SNHL and with abnormal T1-weighted $+\mathrm{C}$ or FLAIR hyperintense signal were GBS (38.5\%), E coli (23.1\%), and S pneumoniae (23.1\%) in our cohort.

\section{DISCUSSION}

SNHL is a serious complication and sequela of bacterial meningitis. The pathogenesis of SNHL from bacterial meningitis is from spread of infection into the inner ear through the cochlear aqueduct and subsequent development of labyrinthitis. ${ }^{8}$ We believe that the detection of abnormal enhancement within the inner ear on MR imaging is secondary to the inflammation and bloodbrain barrier breakdown while the FLAIR hyperintense signal reflects the accumulation of abnormal proteinaceous fluid in the inner ear. Previously reported risk factors for SNHL due to meningitis include $S$ pneumoniae, decreased CSF glucose, increased CSF protein, seizure, and a long hospital duration., ${ }^{5,13}$

Twenty-eight patients (24.3\%) developed SNHL in our cohort. The most common pathogens in our SNHL cohort were GBS (21.4\%) and E coli (29.6\%). In addition, the most common pathogens in patients with SNHL and with abnormal T1-weighted $+\mathrm{C}$ or FLAIR hyperintense signal were GBS (38.5\%) and E coli (23.1\%). GBS and $E$ coli are the 2 most common bacteria causing neonatal meningitis. ${ }^{14}$ The relatively low mean age of our patient population of 50.6 days likely explains the reason the most common pathogens in our cohort were GBS and E coli.

In this large study of infants with bacterial meningitis, we demonstrate high specificity ( $96 \%$ and $94 \%$ ) but average sensitivity (61\% and 50\%) for abnormal enhancement and abnormal FLAIR hyperintense signal, respectively, for predicting SNHL among infants with meningitis. Most (81\%) inner ear enhancement on postcontrast T1-weighted imaging was subjectively graded as mild indicating the radiologist could miss this finding without direct attention to the inner ear. There was excellent agreement between readers and the individual readings were as high as consensus readings, indicating a high likelihood of the reproducibility of these findings in the clinical setting.

Only a few studies are available in the medical literature for comparison with our results. Kopelovich et $\mathrm{al}^{9}$ evaluated T1-weighted $+\mathrm{C}$ MR imaging sequences in 23 children ( 3 months- 14 years) compared with audiometric testing. Only 35\% of their cohort developed SNHL and the authors found $87 \%$ sensitivity and $100 \%$ specificity for contrast enhancement to predict SNHL. ${ }^{9}$ van Loon et $\mathrm{al}^{10}$ evaluated T1weighted $+\mathrm{C}$ and T2-weighted MRIs of 17 patients (3 months77 years) with audiometric data and found inner ear enhancement in $87 \%$ of the ears affected by SNHL. Enhancement of the labyrinth showed $62 \%$ sensitivity and $90 \%$ specificity. ${ }^{10}$ Differences in the 


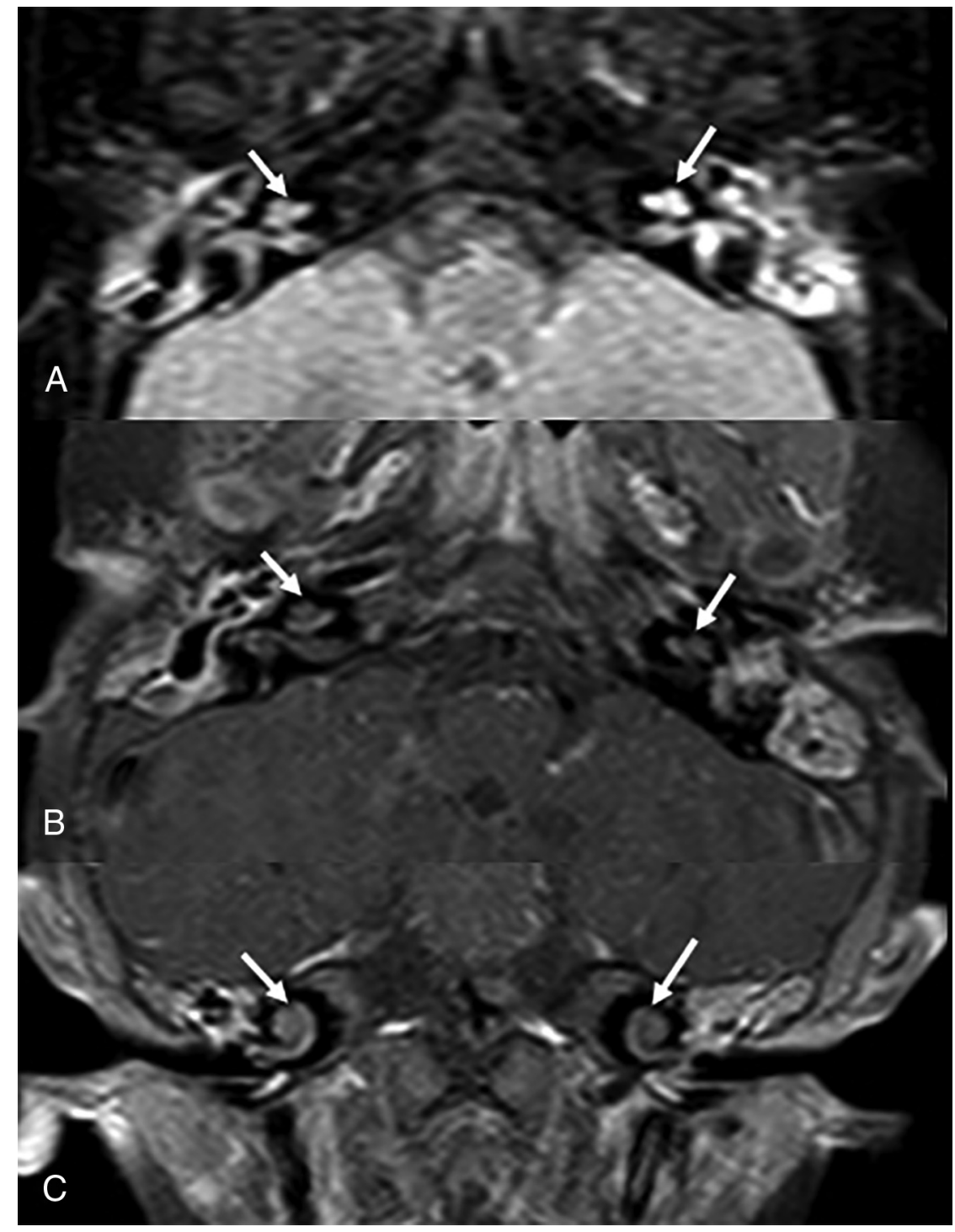

FIG 3. Seven-month-old girl with history of meningitis from $N$ meningitidis. (A) Magnified axial FLAIR, $(B)$ axial Tl-weighted $+C$, and $(C)$ coronal T1-weighted $+C$ images demonstrate abnormal hyperintense FLAIR signal and enhancement of both inner ears. There is an increased enhancement on the mastoids bilaterally. The patient developed bilateral SNHL on follow-up.

low number of postcontrast FLAIR sequences in our data base, we did not include postcontrast FLAIR evaluations in our study design.

Strengths of this study include the large number of patients, a relatively homogeneous age of patients who are most commonly affected by bacterial meningitis, assessment of both a noncontrast and postcontrast sequence for detecting SNHL, and the use of audiometric testing as the criterion standard for diagnosis of SNHL.

Limitations of this study are because of the retrospective nature of the study, and include a discrepancy between number of MRIs and infants, and single-center evaluation of patients with meningitis. Another potential limitation of this study is the subjectivity in the determination of individual MR imaging findings. This subjectivity was mitigated by independent imaging reviews by 2 pediatric neuroradiologists, the consensus diagnosis in discordant findings, and calculation of interobserver agreement. We observed excellent interobserver agreement for both the inner ear enhancement and FLAIR hyperintensity, indicating that the subjectivity in imaging interpretation does not appear to be a significant factor. Because this was a retrospective study, we evaluated the inner ear on wholebrain MR imaging FLAIR and T1weighted $+\mathrm{C}$ sequences, which were available in all patients and had $3-4 \mathrm{~mm}$ section thickness. Thinner section thickness imaging through the temporal bones may improve the sensitivity of detection by providing more images

results of this study and these previous 2 studies may include the patient population (younger age and larger number of patients), and most pathogens being GBS and E coli. Our cohort consists of the most homogeneous and largest patient group to date compared with similar studies. ${ }^{8-10}$ Last, similar to Kutz et al, ${ }^{5}$ we found significantly lower CSF glucose and higher CSF protein in our SNHL cohort to be associated with greater likelihood of abnormal appearance of the inner ear on MR imaging (Table 2).

We did not find any similar studies to compare our abnormal FLAIR hyperintensity results. However, there is 1 report of an adult pneumococcal meningitis patient who developed SNHL and who underwent 3D FLAIR MR imaging that revealed hyperintensity and contrast enhancement in the cochlea and the vestibulum. ${ }^{15}$ Another study among adults reported that 3D FLAIR hyperintensity was positively associated with pretreatment SNHL and presence of vertigo in idiopathic sudden SNHL. ${ }^{16}$ Due to the and higher resolution of the inner ear. In addition, this limitation affected our ability to perform a more detailed inner ear site-specific analysis of the location of the abnormality, but future studies with thinner and higher resolution imaging may be useful for further differentiation. Regardless, the increased recognition provided from this study regarding the diagnostic accuracy of MR imaging for SNHL in these infants may help radiologists more closely assess the inner ear when routine MRIs of the brain are ordered, routinely report on the status of the inner ear in patients with bacterial meningitis, or modify imaging protocols to provide higher resolution imaging of the inner ear in these patients.

\section{CONCLUSIONS}

Abnormal enhancement on T1-weighted $+\mathrm{C}$ MR imaging and abnormal FLAIR hyperintense signal of the inner ear are highly specific for predicting SNHL in infants with bacterial meningitis. 
Table 2: Associated findings for postcontrast T1, FLAIR, and combined evaluation in patients with SNHL $(P<.05)$

\begin{tabular}{lcc}
\hline & $\begin{array}{c}\text { Normal MR Imaging } \\
\text { Findings Mean (SD) }\end{array}$ & $\begin{array}{c}\text { Abnormal MR Imaging } \\
\text { Findings Mean (SD) }\end{array}$ \\
\hline Inner ear enhancement on T1+C & & \\
Age at presentation (days) & $95.2(116.6)$ & $93.4(93.5)$ \\
Time from presentation to MR imaging (days) & $14.1(11.4)$ & $10.1(6.4)$ \\
CSF WBC count & $11692.3(32,494.4)$ & $2674.8(5440.94)$ \\
CSF glucose & $29(14.3)$ & $18.1(11)$ \\
CSF protein & $309.8(236.9)$ & $505.5(216.1)$ \\
FLAIR hyperintensity & & .9 \\
Age at presentation (days) & $74.1(105.1)$ & .3 \\
Time from presentation to MR imaging (days) & $12.9(10.7)$ & $98.2(95.5)$ \\
CSF WBC count & $8613.9(27,966.7)$ & $10(6.6)$ \\
CSF glucose & $35.5(22.4)$ & $2843.62(5624.81)$ \\
CSF protein & $301.5(224.1)$ & $17.9(11.4)$ \\
Combined evaluation & & $498.54(223.3)$ \\
Age at presentation (days) & $95.2(116.6)$ & .04 \\
Time from presentation to MR imaging (days) & $14.1(11.4)$ & $93.4(93.5)$ \\
CSF WBC count & $11,692.3(32,494.4)$ & $10.1(6.4)$ \\
CSF glucose & $29(14.3)$ & $2674.8(5440.94)$ \\
CSF protein & $309.8(236.9)$ & $18.1(11)$ \\
\hline
\end{tabular}

Disclosures: Jesus G. Vallejo—UNRELATED: Royalties: Wolters Kluwer - UpToDate.

\section{REFERENCES}

1. Ku LC, Boggess KA, Cohen-Wolkowiez M. Bacterial meningitis in infants. Clin Perinatol 2015;42:29-45 CrossRef Medline

2. Ouchenir L, Renaud C, Khan S, et al. The epidemiology, management, and outcomes of bacterial meningitis in infants. Pediatrics 2017;140: e20170476 CrossRef

3. de Louvois J, Halket S, Harvey D. Neonatal meningitis in England and Wales: sequelae at 5 years of age. Eur J Pediatr 2005;164:730-34 CrossRef Medline

4. Koomen I, Grobbee DE, Roord JJ, et al. Hearing loss at school age in survivors of bacterial meningitis: assessment, incidence, and prediction. Pediatrics 2003;112:1049-53 CrossRef

5. Kutz JW, Simon LM, Chennupati SK, et al. Clinical predictors for hearing loss in children with bacterial meningitis. Arch Otolaryngol Head Neck Surg 2006;132:941-45 CrossRef Medline

6. Richardson MP, Reid A, Tarlow MJ, et al. Hearing loss during bacterial meningitis. Arch Dis Child 1997;76:134-38 CrossRef Medline

7. Wellman MB, Sommer DD, McKenna J. Sensorineural hearing loss in postmeningitic children. Otol Neurotol 2003;24:907-12

8. Beijen J, Casselman J, Joosten F, et al. Magnetic resonance imaging in patients with meningitis induced hearing loss. Eur Arch Otorhinolaryngol 2009;266:1229-36 CrossRef

9. Kopelovich JC, Germiller JA, Laury AM, et al. Early prediction of postmeningitic hearing loss in children using magnetic resonance imaging. Arch Otolaryngol Head Neck Surg 2011;137:441-47 CrossRef Medline

10. van Loon MC, Hensen EF, de Foer B, et al. Magnetic resonance imaging in the evaluation of patients with sensorineural hearing loss caused by meningitis: implications for cochlear implantation. Otol Neurotol 2013;34:845-54 CrossRef Medline

11. Kestenbaum LA, Ebberson J, Zorc JJ, et al. Defining cerebrospinal fluid white blood cell count reference values in neonates and young infants. Pediatrics 2010;125:257-64 CrossRef

12. Chávez-Bueno S, McCracken GH. Jr. Bacterial meningitis in children. Pediatr Clin North Am 2005;52:795-810 CrossRef Medline

13. Jaremko JL, Moon AS, Kumbla S. Patterns of complications of neonatal and infant meningitis on MRI by organism: a 10 year review. Eur J Radiology 2011;80:821-27 CrossRef

14. Kim KS. Current concepts on the pathogenesis of Escherichia coli meningitis: implications for therapy and prevention. Curr Opin Infect Dis 2012;25:273-78 CrossRef Medline

15. Hara N, Yunoki T, Kubo S, et al. [Pneumococcal meningitis with accompanying severe hearing loss: 3D-FLAIR imaging of the inner ear and treatment]. Rinsho Shinkeigaku 2015;55:119-22 CrossRef Medline

16. Berrettini S, Seccia V, Fortunato S, et al. Analysis of the 3-dimensional fluid-attenuated inversion-recovery (3D-FLAIR) sequence in idiopathic sudden sensorineural hearing loss. JAMA Otolaryngol Head Neck Surg 2013;139:456-64 CrossRef Medline 